\title{
Measurement Algorithm of Clock Jitter Based on Sequential Equivalent Sampling
}

\author{
Feijiang Huang ${ }^{1}$, Binxia $\mathrm{Du}^{2}$, Yong $\mathrm{Cao}^{2}$ and $\mathrm{Gun}^{2}{ }^{2}$,* \\ ${ }^{1}$ Department of Electronic Information and Electrical Engineering, Changsha \\ University, Changsha, 410022, China \\ ${ }^{2}$ School of Aeronautics and Astronautics, University of Electronic Science and \\ Technology of China, Chengdu, 611731, China \\ ligun@uestc.edu.cn
}

\begin{abstract}
For the problem that general characteristic impedance measurement instrument is difficult to measure the size of clock jitter, this paper proposes a measurement algorithm to achieve clock jitter based on sequential equivalent sampling. First, according to the relationship between the equivalent sampling signal and the average signal, we count up the probability density distribution of the clock jitter and use the Tailfit jitter separation techniques to decompose total jitter (TJ) into deterministic jitter (DJ) and random jitter $(R J)$, then count up the peak-to-peak values of periodic jitter (PJ), cycle-to-cycle jitter $(C C J)$ and time interval error (TIE) to achieve a precise measurement of clock jitter. Finally we use the test results of jitter analysis software of Tektronix's TDSJIT3 as a reference to verify the effectiveness of the algorithm. This algorithm provides quantitative analysis means for the stability of characteristic impedance measurement instrument, and provides the reference basis for self-calibration circuit design of subsequent hardware.
\end{abstract}

Keywords: Characteristic impedance, Sequential equivalent sampling, Clock jitter, Jitter separation

\section{Introduction}

With the development of electronic information industry, the industry market of printed circuit board (PCB) has strong demand, due to intuitively reflect the changes of characteristic impedance with the PCB traces, the instrument which can test PCB characteristic impedance has been widely used [1-2]. The characteristic impedance is transient impedance which can reflect the characteristics of PCB transmission line [3], Since the frequency of clock timing and data transfer speed are getting higher and higher, these factors, such as the rise time speed of step signal, the quality of step pulse, the stability of the clock and the noise of the instrument caused by impedance mismatching can affect the performance and index of the system, eventually they will seriously affect the signal quality [4]. Therefore, the control of characteristic impedance is becoming increasingly stringent. Jitter has a very important impact for the stability and performance of the instrument [5], for characteristic impedance measurement instrument, the clock jitter is an important factor which can affect the sampling results. Clock controls the instrument's sampling time, and any clock jitter will cause deviation of sampling time, result in errors between the acquired signal and the original signal, this can affect the whole signal integrity and noise ratio, ultimately restrict the performance of the instrument [6-8].

Jitter is the unpredictable timing variations of the signal's single edge, it refers to the deviation of the adjacent side edge or a non-adjacent edge with the ideal position (time or phase), generally it is represented by the unit of picoseconds (ps) or the unit interval (UI)[9]. The existing papers have done a lot of analysis on clock jitter measurements,

*Corresponding Author: Gun Li, E-mail: ligun@uestc.edu.cn 
paper [10] established a histogram of the acquired points of samples, each number of the ordinate of the histogram divided by the total number of acquired samples, and used the height values of the rectangle to represent the probability that the sampling data appears in its corresponding abscissa within this range. When the width value of the rectangle is becoming small, the outline of the histogram tends to become a continuous curve, which can obtain a probability density function of jitter; Since the jitter histogram analysis method is based on statistics, we can not figure out the relationship between time and jitter and characteristics of jitter cycle from the jitter histogram, and the use of time trend analysis method can visually display the relationship between jitter and time of collected samples [11, 12]; The paper[13] through cumulative superposition of the collected signal bit, obtained the eye diagram which contains a large number of information that can reflect the overall statistical characteristics of the signal, then according to the measured size of the "eye" opening extent to quickly determine signal quality of eye, and assess the merits of the system. When the jitter is violent, the opening extent of 'eye' will be smaller; The paper [14] through the fourier transform to transform the collected signal from time domain into the frequency domain for analysis to obtain a relationship diagram signal between the frequency and the amplitude in the frequency domain, then separated the deterministic jitter components and random jitter components of the frequency spectrum. In this paper, the key techniques of clock jitter measurement in characteristic impedance measurement instrument software have been studied to achieve measurement algorithm of clock jitter based on sequential equivalent sampling, which can measure clock jitter with higher accuracy.

\section{Jitter Model and Jitter Separation Method}

\subsection{Jitter Model}

Total jitter (TJ) can be decomposed into deterministic jitter (DJ) and random jitter (RJ), deterministic jitter (DJ) can be further decomposed into periodic jitter (PJ), datadependent jitter (DDJ), DDJ also can be decomposed into inter-symbol interference (ISI), and duty cycle jitter (DCDJ) [15].

Random jitter is a kind of unpredictable jitter caused by random noise, random noise can be arbitrary distribution theoretically. In this model, the random jitter is regarded as the Gaussian distribution, the sampling values of the samples which adhere to Gaussian distribution mostly concentrate in the vicinity of the average value, but there are some individual sample values which deviate from the mean value, the peak-to-peak value among the sample values can be infinite. Therefore, random jitter can only be quantitative analyzed from the perspective of the mean value $\mu$ and mean square value $\sigma$.

Deterministic jitter is bounded, it has the characteristics of repeatability and predictability, its limited peak-to-peak value can be decomposed and estimated by the jitter separation techniques based on the sample data obtained from experiments. Periodic jitter (PJ) refers to the change situation of clock signal or data signal with the cycle, you can describe it through cycle-cycle jitter (CCJ) and time interval error (TIE) [9]. CCJ is the results through counting and testing the difference of adjacent clock or data cycle, it can be obtained by calculating the difference between the adjacent period jitter. TIE is the difference of the time between the actual edge transitions and ideal edge transitions where the signal is carrying out level conversion. Because PJ, CCJ and TIE are all the methods of describing jitter, you can get the other two values when measuring one of the PJ, CCJ and TIE values. The relationship of the three values is shown in formula (1), (2) and (3).

$$
\begin{array}{r}
C C J_{i}=P_{i+1}-P_{i}=P J_{i+1}-P J_{i} \\
P J_{i}=T I E_{i}-T I E_{i-1}
\end{array}
$$




$$
T I E_{i}=\sum_{j=1}^{i} P J_{j} \quad(1 \leq j \leq i)
$$

\subsection{Jitter Separation Method}

Jitter separation decomposes the measured total jitter into different components and estimates the size of each component, infers the main reasons for jitter generation, and then solves them. Because the amplitude of RJ and DJ does not have obvious boundaries, the FFT method is simple, but it has limited accuracy; Time lag correlation (TLC) algorithm completes the jitter separation by solving equations of the correlation function between jitter components. However, the algorithm will make non-zero point of the overall jitter generate burrs phenomenon. In this paper, a computational algorithm with high accuracy of Tailfit is used to complete jitter separation [16], but the results of the algorithm are affected by the number of statistical samples, and only in the case of a sufficient number of samples, we can obtain accurate results. Tailfit algorithm assumes that DJ and RJ are independent and mutually uncorrelated [17,18], TJ, DJ and RJ satisfy the equation (4), which can obtain the total jitter by the convolution of DJ and RJ, total jitter retains the characteristics of RJ, so in the case of knowing the distribution of TJ, according to the algorithm, we can fit the trailing portion of TJ, thus get the distribution of RJ. DJ and RJ can be calculated as formula (5) and (6).

$$
\begin{array}{r}
P_{T J}(x)=P_{R J}(x) * P_{D J}(x) \\
R J=(\sigma L+\sigma R) / 2 \\
D J=x_{-} \text {Right }-x_{-} \text {Left }
\end{array}
$$

\section{Clock Jitter Measurement Algorithm based on Sequential Equivalent Sampling}

\subsection{The Principle of Sequential Equivalent Sampling}

Sampling method using in sampling system of characteristic impedance measurement instrument is sequential equivalent sampling, the principle of sequential equivalent sampling is shown in Figure 1 [19]. In figure 2, $A(t)$ represents the original signal of period $T$, assuming that triggers sampling signal of $S(t)$ at the arrival of wave trough of $A(t)$ each time. After a delay time of $\Delta t$, the original signal is sampled by $S(t)$, delay time of the first sampling is $\Delta t$, the second sampling delay time is $2 \Delta t$, and so on. So that points collected from $A(t)$ are $A(T+\Delta t), A(2 T+2 \Delta t), A(3 T+3 \Delta t)$, etc. Those points are arranged in sampling sequence, then the waveform of $A_{s}(t)$ is obtained, sampling period of $A_{s}(t)$ is equivalent to $T_{s}=\Delta t$, while the actual sampling interval is $T_{s}=T+\Delta t$, thus achieving a sequential equivalent sampling.

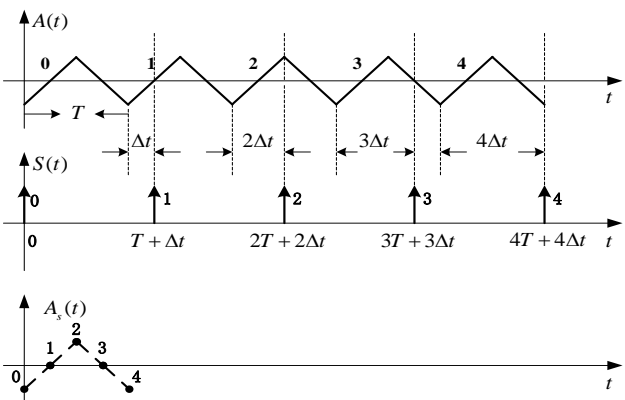

Figure 1. Schematic Diagram of Sequential Equivalent Sampling 


\subsection{Clock Jitter Measurement Algorithm Based on the Principle of Sequential Equivalent Sampling}

3.2.1 The Principle of Algorithm: Assuming that ideal signal inputted to the instrument is $x_{a}(t)$, at sampling time $t_{k}(k=0,1,2,3 \cdots)$, the ideal value of $x_{a}(t)$ should be $x_{a}\left(t_{k}\right)$, but in actual conditions due to the presence of clock jitter $\Delta t_{k}$, At the moment $t_{k}$ collected signal value drift up and down at $x_{a}\left(t_{k}\right)$, resulting in an error of $e_{k}$ in the amplitude. Assuming the probability density distribution of clock jitter is $P(\Delta t)$, then via $\mathrm{N}$ times collection at the moment $t_{k}$ and averaged, to get the formula (7).

$$
\overline{x_{a}\left(t_{k}\right)}=E\left(x_{a}\left(t_{k}\right)\right)=\lim _{N \rightarrow \infty} \frac{1}{N} \sum_{k=0}^{N-1} x_{a}\left(t_{k}-\Delta t_{k}\right) P\left(\Delta t_{k}\right)=\int_{-\infty}^{\infty} x_{a}\left(t_{k}-\tau\right) p(\tau) d \tau
$$

Wherein, $\overline{x_{a}\left(t_{k}\right)}$ is the average value of $x_{a}\left(t_{k}\right), \tau$ is the integration variable. By the formula (7) shows that, for an arbitrary moment $t$ has the formula (8).

$$
\overline{x_{a}(t)}=\int_{-\infty}^{\infty} x_{a}(t-\tau) p(\tau) d \tau
$$

$x_{a}(t)$ and $\overline{x_{a}(t)}$ are quantified as $x[l]$ and $\overline{x[l]}$ after sequential equivalent sampling, as can be known based on the principle of sequential equivalent sampling, $x[l]=x_{a}\left(l T_{e}\right)$, $\overline{x[l]}=\overline{x_{a}\left[l T_{e}\right]}$, where $T_{e}$ is the sequential equivalent sampling time interval, so that $t=l T_{e}$, the equation (8) turn to equation (9).

$$
\overline{x[l]}=\int_{-\infty}^{\infty} x_{a}\left(l T_{e}-\tau\right) p(\tau) d \tau
$$

The probability density function $p(\tau)$ of clock jitter becomes to $p[n]=p\left[n T_{e}\right]$ in accordance with the principles of sequential equivalent sampling, $p(\tau)$ is jitter value at the moment $n T_{e}, p(\tau)$ can use the unit impulse function $\delta(t)$ to express as Equation (10).

$$
p(\tau)=\sum_{n=-\infty}^{\infty} p[n] \delta\left(\tau-n T_{e}\right)
$$

Formula (11) can be obtained by combining (9) and (10) .

$$
\overline{x(l)}=\int_{-\infty}^{\infty} x_{a}\left(l T_{e}-\tau\right) \sum_{n=-\infty}^{\infty} p[n] \delta\left(\tau-n T_{e}\right) d \tau=\sum_{n=-\infty}^{\infty} x[l-n] p[n]
$$

Since in the actual case $p[n]$ is a finite-length sequence, so the sequence of the clock jitter can be expressed as Equation (12), then the equation (11) becomes equation (13).

$$
\begin{aligned}
& p[l]=\sum_{n=1}^{N} p[n] \delta[l+1-n] \\
& \overline{x(l)}=\sum_{n=1}^{N} x[l+1-n] p[n]
\end{aligned}
$$

Formula (13) is the jitter required to solve, the formula (13) expressed in matrix form as equation (14).

$$
\overline{\mathbf{X}}=\mathbf{X P}
$$

Assuming collected signal is sequence $\mathbf{X}=[x[1], x[2] \cdots, x[M]]$ containing the length of M points, $p[n]$ is sequence $\mathbf{P}=[p[1], p[2] \cdots, p[N]]$ containing the length of $\mathbf{N}$ points, and $M \geq N, \overline{\mathbf{X}}$ is sequence $\overline{\mathbf{X}}=[\overline{x[1]}, \overline{x[2]} \cdots, \overline{x[L]}]$ containing the length of $\mathrm{L}$ points, the subscript of the three sequences $\mathbf{X}, \mathbf{P}$ ang $\overline{\mathbf{X}}$ are starting from 1, expanding Formula (14) can obtain Formula (15). 


$$
\left[\begin{array}{c}
\overline{x[1]} \\
x[2] \\
\overline{x[3]} \\
\vdots \\
\overline{x[L]}
\end{array}\right]=\left[\begin{array}{cccc}
x[1] & 0 & \cdots & 0 \\
x[2] & x[1] & \cdots & 0 \\
\vdots & \vdots & \ddots & \vdots \\
x[N] & x[N-1] & \cdots & x[1] \\
\vdots & \vdots & \ddots & \vdots \\
x[M] & x[M-1] & \cdots & x[M-N+1] \\
0 & x[M] & \cdots & x[M-N+2] \\
0 & 0 & \ddots & \vdots \\
0 & 0 & \cdots & x[M]
\end{array}\right]\left[\begin{array}{c}
p[1] \\
p[2] \\
\vdots \\
p[N]
\end{array}\right]
$$

In the formula (15), according to the definition of convolution can be seen $L=M+N-1, \mathbf{X}$ is Toeplitz matrix of $\mathrm{L} \times \mathrm{N}$ order, there is no solution for the equation, solving the equation by the least squares method and calculating as of formula (16).

$$
\mathbf{P}=\left(\mathbf{X}^{T} \mathbf{X}\right)^{-1}\left(\mathbf{X}^{T} \overline{\mathbf{X}}\right)
$$

Wherein, $\mathbf{X}^{T}$ represents a transposed matrix of $\mathbf{X}$. Solution of equation (16) is related with the matrix of $\mathbf{Y}=\mathbf{X}^{T} \mathbf{X}$ whether it is reversible matrix (singular matrix), when $\mathbf{Y}$ is a singular matrix, the equation has no solution, there is in the respect of minimum variance, equation has no solution. In this case, we can only find approximate solutions of equation group, by adding a small amount of matrix in the formulas, making Y become a singular matrix, thereby obtaining an approximate solution of equation group, calculation formulas are shown in formula (17).

$$
\mathbf{P}=\left(\mathbf{X}^{T} \mathbf{X}+\varepsilon^{2} \mathbf{E}\right)^{-1}\left(\mathbf{X}^{T} \overline{\mathbf{X}}\right)
$$

Wherein, $\mathbf{E}$ is the unit matrix having the same row and column with the matrix $\mathbf{Y}$. In order to ensure the accuracy of $\mathbf{P}$, in the formula (17) the value of $\varepsilon^{2}$ should be similar to the error caused by noise in the quantization process (quantization error or quantization noise), the quantization error represents the difference between the input and output signals, it is obtained based on a large number of statistics[20,21]. Due to the characteristic impedance tester using the quantization approach of "directed rounding"when sampling and quantifying, assuming that the quantization unit is $q$, the quantization error is a random variable uniformly distributed between $[-q / 2, q / 2]$, so the mean of the quantization error is 0 , the root of mean square is $\sigma=q / \sqrt{12}$. Assuming the quantization error is $x_{k}$, after transformed into the frequency domain the sequence of its is $X_{m}$, and $k=0,1, \cdots N-1, m=0,1, \cdots N-1$, formula (18)can be obtained according to energy conservation theorem of parseval .

$$
\sum_{k=0}^{N-1}\left|x_{k}^{2}\right|=\frac{1}{N} \sum_{m=0}^{N-1}\left|X_{m}^{2}\right|
$$

Since the quantization error is $N q^{2} / 12$, value of $\varepsilon^{2}$ should be smaller than the quantization error, as shown in formula (19).

$$
\varepsilon^{2} \leq \frac{1}{N} \sum_{m=0}^{N-1}\left|X_{m}{ }^{2}\right|=\sum_{k=0}^{N-1}\left|x_{k}^{2}\right|=\frac{N q^{2}}{12}
$$

After collecting the data of clock jitter, you can calculate the distribution of the total jitter based on the relationship between data of clock jitter and its average data,then use jitter separation methods of Tailfit separate the overall jitter (TJ) into deterministic jitter (DJ)and random jitter (RJ) two parts . Since the cycle of actual clock signal averaged is approximately equal to the cycle of ideal clock signal, therefore we can calculate the cycle jitter of PJ and do not require to reference the clock, then the three jitter values of PJ, CCJ 
and TIE can be calculated by equation (1), (2) and (3) to get statistical values of TJ, DJ, $\mathrm{RJ}, \mathrm{PJ}$, and TIE,CCJ in clock jitter .

\subsubsection{Algorithm Procedure:}

(1) Getting clock signals $\mathbf{X}$ collected from multiple sets, assuming that $\mathbf{X}$ is a sequence of $\mathrm{M}$ points, jitter of $p[n]$ is a sequence of $\mathrm{N}$ points, in order to obtain the jitter for each data point, we must make the values of $\mathrm{M}$ equal to the values of $\mathrm{N}$. Handling each set of data to obtain a sequence $\overline{\mathbf{X}}=[\overline{x[1]}, \overline{x[2]} \ldots, \overline{x[L]}]$ of L points , the data for each point in front of the $\mathrm{N}$ in $\overline{\mathbf{X}}$ is obtained by doing average for the data of subsequent $\mathrm{N}$ points in signal $\mathbf{X}$, In $\overline{\mathbf{X}}$ from the $\mathrm{N}+1$-th points to the L-th point, there are obtained by doing average for the data of previous $\mathrm{N}$ points in signal $\mathbf{X}$, the data which is not exist

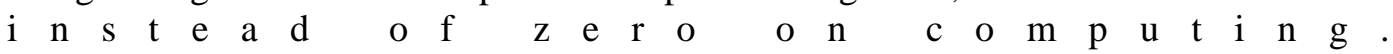

(2) Determining whether $\mathbf{Y}=\mathbf{X}^{T} \mathbf{X}$ is singular matrix, that is when the value of determinant of $\mathbf{Y}$ is $0, \mathrm{~W}$ is a singular matrix, otherwise it is a non-singular matrix.

(3) If $\mathbf{Y}$ is a non-singular matrix, then $\mathrm{Y}$ has the inverse matrix. According to the formula $\mathbf{P}=\left(\mathbf{X}^{T} \mathbf{X}\right)^{-1}\left(\mathbf{X}^{T} \overline{\mathbf{X}}\right)$, we can calculate the value of $\mathrm{P}$, where $\mathrm{P}$ is the sequence with $\mathrm{N}$ points. If $\mathbf{Y}$ is a singular matrix, then $\mathrm{Y}$ doesn't have the inverse matrix. In this case, according to the formula $\mathbf{P}=\left(\mathbf{X}^{T} \mathbf{X}+\varepsilon^{2} \mathbf{E}\right)^{-1}\left(\mathbf{X}^{\mathbf{T}} \overline{\mathbf{X}}\right)$, we can calculate the value of $\mathrm{P}$, where the scope of $\varepsilon^{2}$ is $\varepsilon^{2} \leq N q^{2} / 12$, due to the quantization unit of characteristic impedance tester is $14 \mathrm{bit}$, where $q=14 \mathrm{bit}$. Then we can calculate the jitter value of each group of data, it is also the value of $P$.

(4) Solving average of the jitter values obtained for each group of data and making the results after average as a total jitter TJ.

(5) Using the method of histogram statistics distribution of TJ, removing abnormal data of too large or too small in measured TJ, the probability density function PTJ of the TJ can be obtained by the ordinate of the histogram divided by the total number of samples. In order to facilitate the tail fitting, we need to use the moving average filter smoothing PTJ of TJ, assuming the number of histogram is Num, the length for Num * $3 \%$ of the moving average filter can get a good smoothing effect.

(6) Based on the probability density function of total jitter, we determine the left and right trailing regions, assuming that there are 600 histograms, the trailing portion of the left side only needs to process the histogram of 300 on the left side, and the right trailing portion only needs to process the histogram of 300 on the right side.

(7) The peak value of right trailing portion denoted Mag_pp, made the average value of the data accounting for 3\% before and after Mag_pp in the total area as the initial value of the average in the right trailing side, denoted Mean_init, and denoted the variance of all the data in the right of the peak as initial variance of Sigma_init intended to be fitting.

(8) Conducting optimization processing on the basis of Mean_init and Sigma_init, causes the value of the fitted Gaussian distribution in a certain neighborhood are coincides with the data of probability density function of TJ, that obtained the minimum mean and variance of test values $\chi^{2}$ as the initial value to fitting the best mean and variance in the next stage, the formula of $\chi^{2}$ such as formula (20) shows below. Wherein, $Y_{H i}$ represents the probability value in the probability density function of TJ, $Y_{G i}$ represents the value to fitted in Gaussian probability distribution , $k$ represents the total data points in test interval of the selected .

$$
\chi^{2}=\sum_{i=1}^{k} \frac{\left(Y_{H i}-Y_{G i}\right)^{2}}{Y_{G i}}
$$


This process is done in two stages, the specific process of the two-stages are the same, all make fitting process on these two parameters of Mean_init and Sigma_init, excepts that selected neighborhood range and step size are different, neighborhood range of the first step is $50 \%$, in steps of $4 \%$, neighborhood of the second step is $12 \%$ in steps of $0.5 \%$.

(9) On the basis of step (8), on the third stage to do a more accurate fitting process, assuming that the initial value of the step size is $0.5 \%$, threshold is $0.001 \%$, by continuously decrease the step size from the initial value, at the same time, for each step size, in the case that unchanged the mean, adjust the the variance, to determine the optimal variance at the current step , and in the same circumstances of variance,, adjusting the mean, in order to determine the optimal mean at the current step size, the specific implementation process, as shown in Figure 2. That means mean of the right side trailing portion is variance of the right side trailing portion. According to the same procedure, we can calculated the fitting mean of the left side trailing portion and the fitting variance of the left side trailing portion

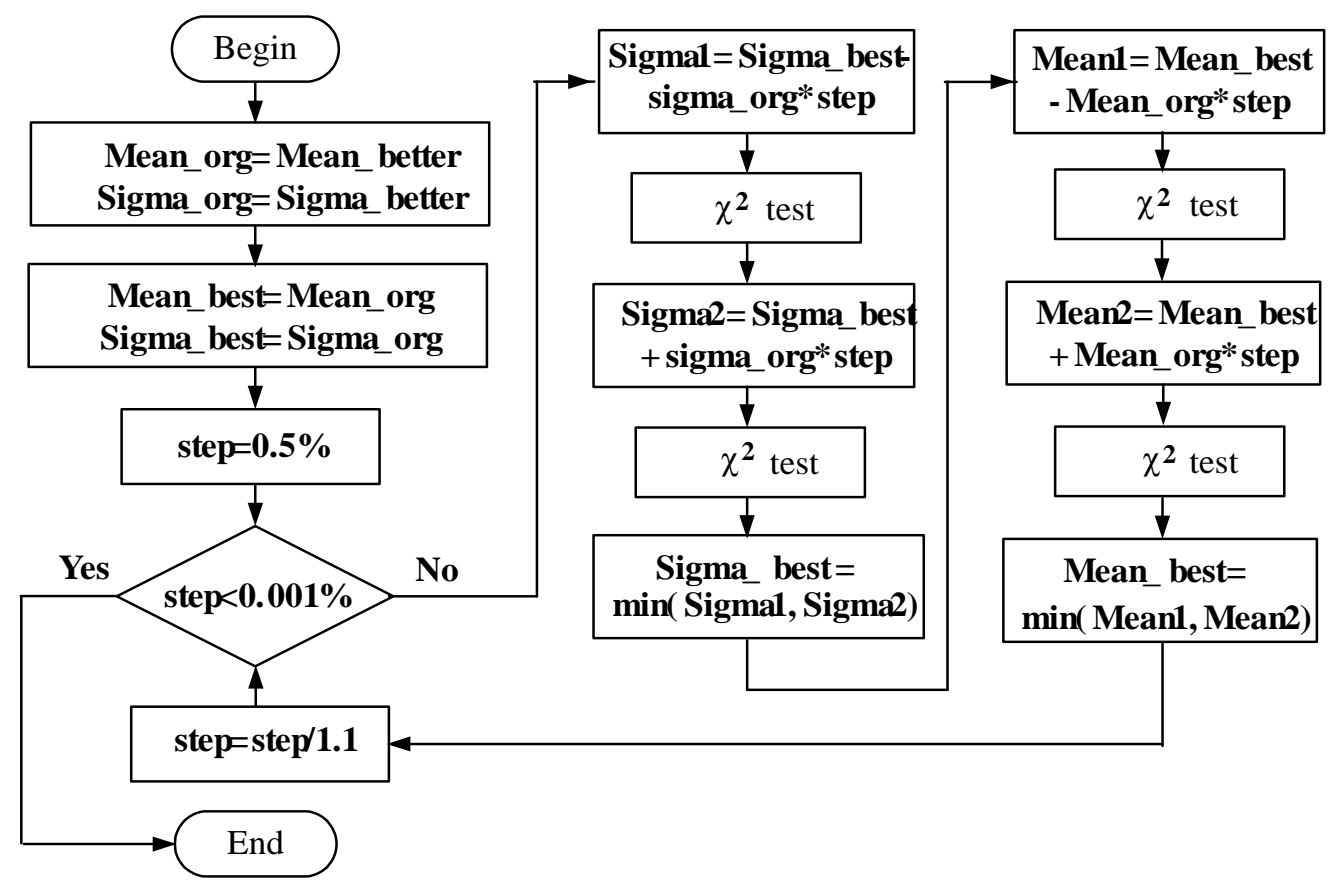

Figure 2. Fitting Flowchart of the Trailing Portion in the Third Phase

(10) Using mean and variance calculated by (9) step of the left trailing regional and the right trailing region, according to the formula $R J=(\sigma L+\sigma R) / 2$ and formula $D J=x_{-}$Right $-x_{-}$Left obtaining the values of $\mathrm{RJ}$ and DJ, enabling the jitter separation.

(11) Finding changes of the cycle jitter of the measured clock signal along with time. First, average processing a group collected clock signals to obtain a clock cycle which is similar to the ideal case, and then calculate the size of each cycle about the clock signal in a single acquisition based on the time algorithm of rising edge, each cycle of the acquired signal subtracted the clock cycle in the ideal case and calculated the jitter cycle of the acquired clock signals, finally according to the relationship among CCJ, TIE and PJ calculated the changes of the peak value of TIE and CCJ along with of times.

\section{Analysis of Measurement Result}

Clock frequency of characteristic impedance tester is $500 \mathrm{MHz}$, the actual sampling interval is $2 \mathrm{~ns}$, equivalent sampling interval is $10 \mathrm{ps}$. Clock signal collected with the jitter 1 
is shown in Figure 3. Due to a lot of data, the figure shows only one part of the data. In experiment collects 1000 waveform of clock signal, each collected clock signal has 5000 cycles. After the averaging process, the result of one of the collected clock signal is shown in Figure 4.

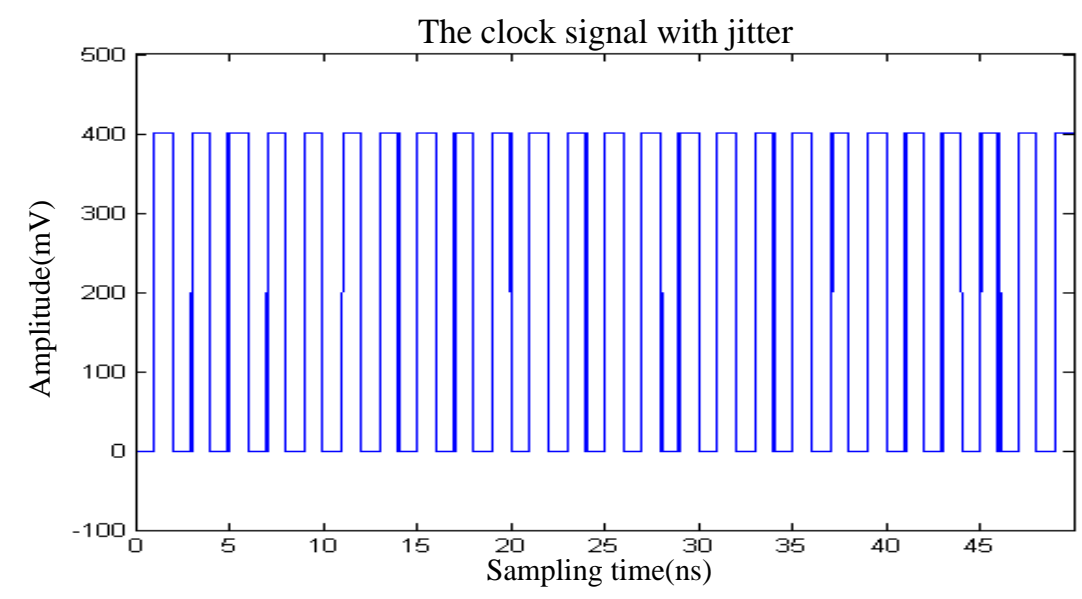

Figure 3. The Collected Clock Signal

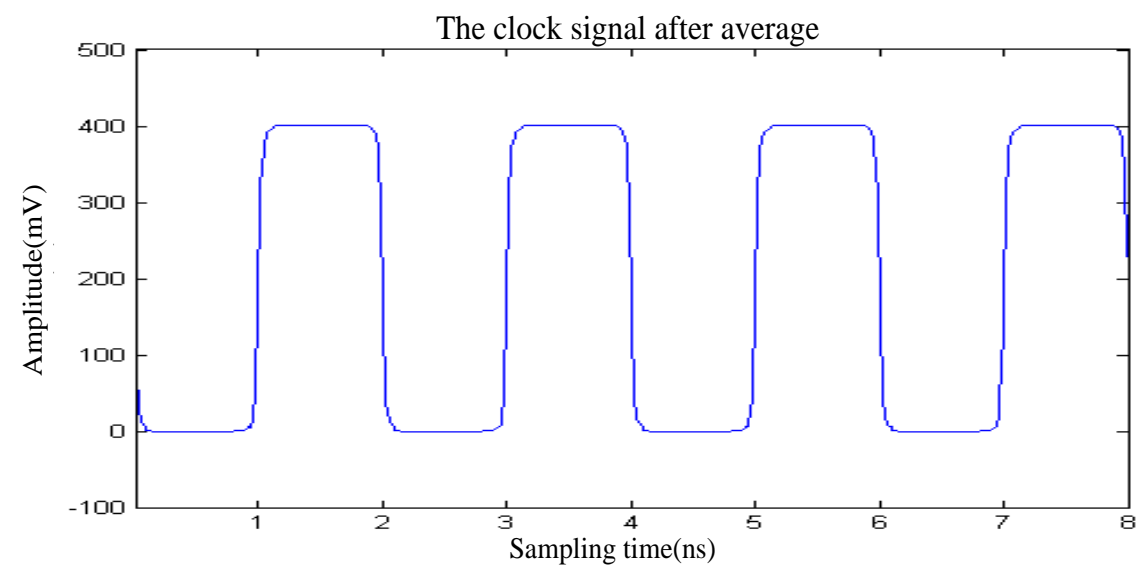

gure 4. Averaged Clock Signal

Calculate the total jitter and obtain a probability density function of TJ through dividing the ordinate of histogram by the total number of samples, fitting results for trailing portions both of the left and right are shown in Figure 5, through the clock jitter algorithm to calculating that $x_{-}$Left is $-30.81 \mathrm{ps}, x_{-}$Right is $29.26 \mathrm{ps}, \sigma L$ is $3.23 \mathrm{ps}$, $\sigma R$ is $2.71 \mathrm{ps}$, mean variance of $\mathrm{RJ}$ is $2.97 \mathrm{ps}$, peak value of $\mathrm{DJ}$ is $59.53 \mathrm{ps}, \sigma L$ and $\sigma R$ are not equal because of the deviation in the process of statistics TJ histogram and it will be affected by the sampling noise in the process of collecting clock signal, As can be seen from Figure 5, peak value of TJ is 160ps, due to TJ is relate with RJ and DJ, RJ satisfy the Gaussian distribution, the most value can theoretically tends to infinity, so its peak value will theoretically tends to infinity, the size of TJ is related with the number of statistical samples, with the more sample size, comes the greater value of TJ. In a statistical process, emergence of maximum and minimum values of TJ is a small probability event, So in the statistical process, as long as number of samples is sufficiently larger, you can use the statistical peak value of TJ to estimate the overall jitter of clock. 


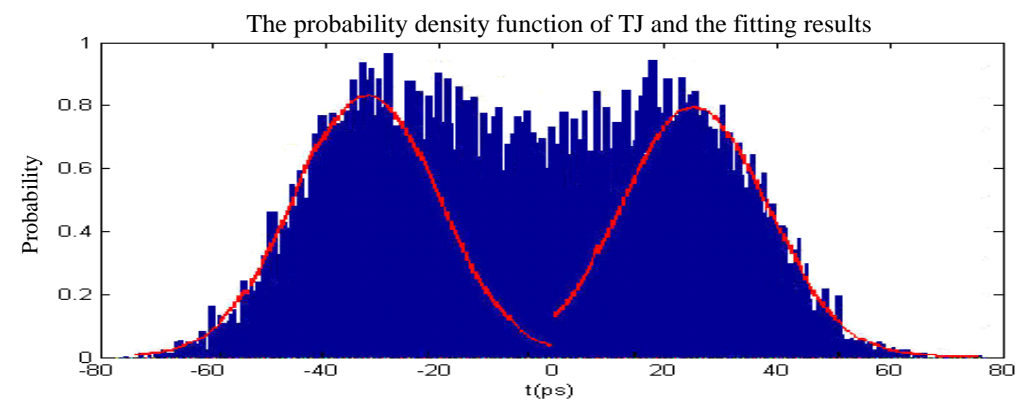

Figure 5. Probability Density Function of TJ and The Fitting Results

Statistical result of PJ is shown in Figure 6, the peak value of PJ calculated out from simulation is $59.84 \mathrm{ps}$, This is substantially consistent with result 59.53ps of DJ calculating by the clock jitter algorithm, as for the clock, deterministic jitter only contains PJ, does not contain data dependent jitter, so DJ and PJ all represent deterministic jitter, only ways of representation and computation are different. The statistical results of CCJ shown in Figure 7, the peak value of CCJ calculated out from simulation is 118.22ps, due to the maximum error of a single period is approximately $30 \mathrm{ps}$, the minimum value is approximately $-30 \mathrm{ps}$, the maximum interval between cycles is approximately 60ps, the minimum value is about $-60 \mathrm{ps}$, so peak values of CCJ is about $120 \mathrm{ps}$. The statistical results of TIE, shown in Figure 8, peak value of TIE calculated from simulation is 397.23ps.

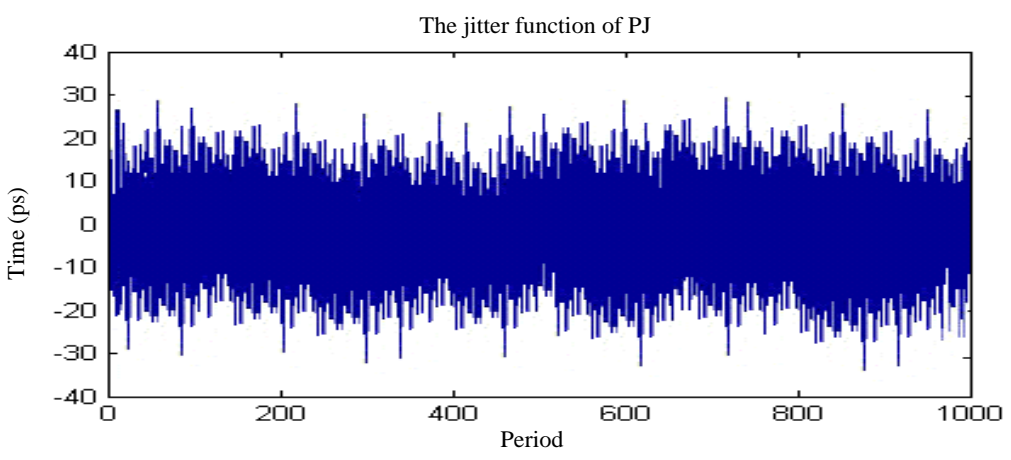

Figure 6. The Statistical Results of PJ

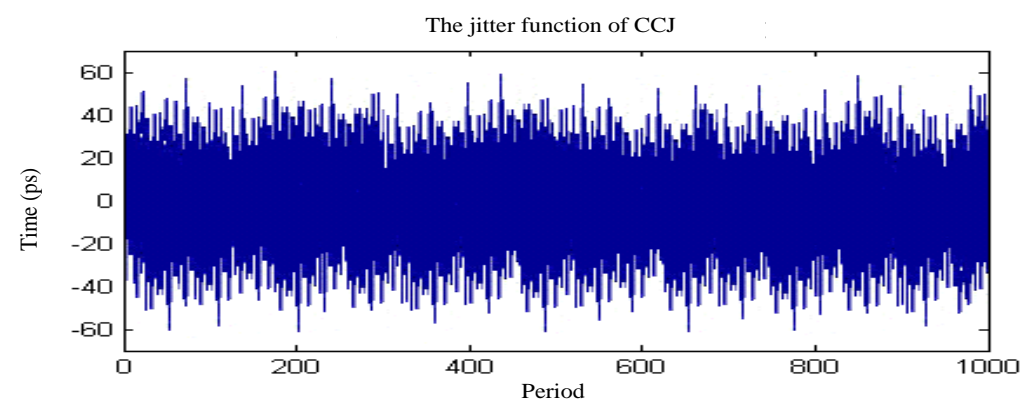

Figure 7. The Statistical Results of CCJ 


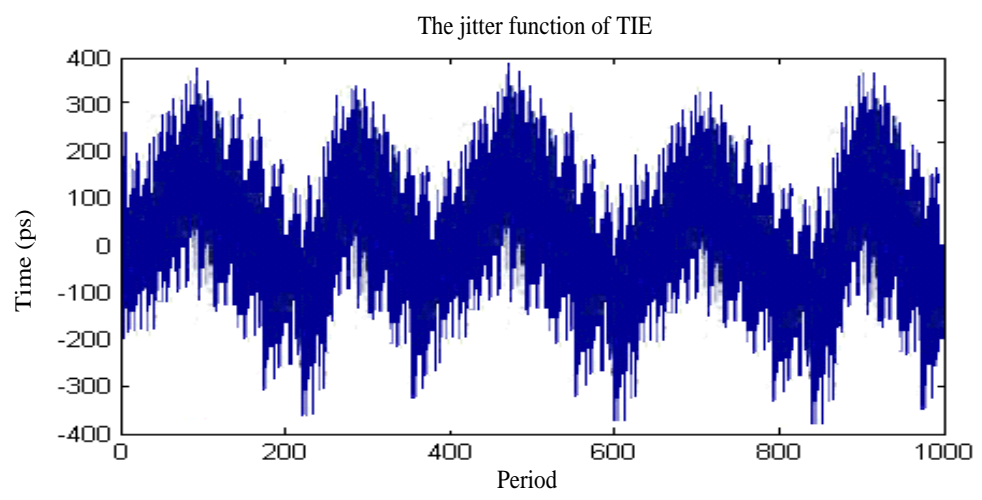

Figure 8. The Statistical Results of TIE

The results calculated by clock jitter algorithm and results tested by Tektronix jitter analysis software TDSJIT3 are shown in Table 1. From the table 1, we can see that the error of clock jitter algorithm and TDSJIT3 test are less than 5\%, then we can estimate that the clock jitter algorithm can achieve the effective measurement for clock jitter.

Table 1. The Test Results Comparison between Clock Jitter Algorithm and TDSJIT3

\begin{tabular}{cccc}
\hline $\begin{array}{c}\text { Jitter } \\
\text { components }\end{array}$ & TDSJIT3(ps) & $\begin{array}{l}\text { Clock jitter } \\
\text { algorithm(ps) }\end{array}$ & Error \\
\hline RJ & 2.93 & 2.97 & $1.41 \%$ \\
DJ & 58.16 & 59.53 & $2.36 \%$ \\
TJ & 168.05 & 160.00 & $-4.49 \%$ \\
PJ & 58.24 & 59.84 & $2.74 \%$ \\
CCJ & 115.48 & 118.22 & $2.37 \%$ \\
TIE & 386.29 & 397.23 & $2.83 \%$ \\
\hline
\end{tabular}

\section{Conclusions}

From the reality that it is difficult to accurately measure clock jitter for the general impedance measurement instrument, this paper studies the separation and measurement method of clock jitter, and proposes a new measurement algorithm of clock jitter based on sequential sampling, the calculation results show that the algorithm can achieve high precision measurement for clock jitter, and compare it with test results from jitter analysis software TDSJIT3, the error of this paper's method is less than 5\%. In the subsequent hardware design of characteristic impedance measurement instrument, according to the jitter value which is obtained from clock jitter algorithm, we can detect the stability of the instrument in real-time. On this basis we can design self-calibration circuit to develop the characteristic impedance instrument with higher stability, and improve the long-term repeatability accuracy of the instrument.

\section{Acknowledgements}

This study was supported by Hunan Provincial Natural Science Foundation of China (No.2015JJ2016, No.11JJ3072), Scientific Research Fund of Hunan Provincial Education Department of China (No.13A115) and Science and Technology Planning Project of Changsha of China (No. K1309019-11). 


\section{References}

[1] T. Wang and J. Bai, "The Analysis on the Foreign Direct Investment's Influence to China PCB Industry's Transformation and Optimization”, Economic Research Guide, vol. 30, (2013).

[2] J. Li, "The Research on Test Method of Characteristic Impedance in PCB", Electronic Science and Technology, vol. 11, (2013).

[3] E. Bogatin, "Signal Integrity: Simplified", Prentice Hall, USA (2004).

[4] R. Ludwig and P. Bretchko, "RF Circuit Design: Theory and Applications (2nd Edition)", Prentice Hall, USA (2009).

[5] L. Li, "Jitter Analysis for High Speed Serial Interconnects", Master Dissertation, Xidian University, China (2009).

[6] M. shinagawa, Y. akazawa and T. wakimoto, "Jitter Analysis of High-speed Sampling Systems" IEEE Journal of Solid-State Circuits, vol. 1, no. 25, (1990)

[7] T. M. Souders, D. R. Flach and C. Hagwood, "The Effects of Timing Jitter in Sampling Systems", IEEE Transaction on Instrument and Measurement, vol. 1, no. 39, (1990).

[8] J. Zaid, A. H. Towfic and H. Sayed, "Clock Jitter Estimation in Noise", IEEE International Symposium on Circuits and System (ISCAS), Rio de Janeiro, Brazil, (2011).

[9] M. Lu, "Implementation of Full Jitter Performance Test in High-speed Serial Links with ATE", The Tenth International Conference on Electronic Measurement \& Instruments, Chengdu, China (2011).

[10] [10] Jin Yadong. Jitter Measurement and Separation Based on Histogram. Master Dissertation, Xidian University, China (2011).

[11] R. Stephens, "Analyzing Jtter at Hgh Data Rates", Communications Magazine, IEEE, vol. 2, no. 42, (2004).

[12] D. Pommerenke, "Jitter: Basics, Relevance and Measurement Methods", IEEE International Symposium on EMC, Detroit, MI, USA (2008)

[13] Tektronix Inc., Eye Diagram and Jitter, Advanced Television Engineering, vol. 1, (2006).

[14] L. Ma, "The Research on Analysis and Decomposition of Jitter", Master Dissertation, Xidian University, China (2011).

[15] A. Kuo, T. Farahmand and N. Ou, "Jitter Models and Measurement Methods for High-speed Serial Interconnects", ITC International Test Conference, USA (2004).

[16] Y. Fan, "Jitter Separation Based on the Tail-Fit Algorithm", Electronic Science and Technology, vol. 2, no. 24, (2011).

[17] M. P. Li, J. Wilstrup and R. Jessen, “A New Method for Jitter Decomposition Through Its Distribution Tail Fitting", International Test Conference. Atlantic City, NJ, (1999).

[18] L. Wang, "Decomposition and Analysis of Jitter in Clock and Data Based on Statistical Domain", Master Dissertation, Xidian University, China (2011).

[19] D. Wang, "The Design and implementation of high speed data acquisition system based on equivalent sampling", Master Dissertation, Changchun University of Technology, China (2010).

[20] W. Gans, "The Measurement and Deconvolution of Time Jitter in Equivalent-time Waveform Samplers", IEEE Transaction on Instrument and Measurement, vol. 1, no. 32, (1983).

[21] Y. Li, S, Zhou and Q. An, "New Method for Accurate Measurement of Clock Jitter Based on Equivalenttime Sampling", System Engineering and Electronics, vol. 4, no. 28, (2006).

\section{Authors}

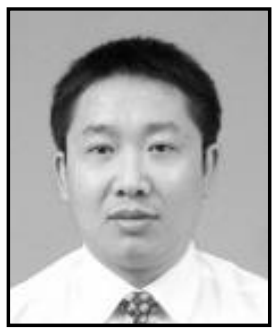

Feijiang Huang, He received his M.S. degree in circuit and system (2003) from Guanxi Normal University and Ph.D. degree in astrometry and celestial mechanics (2009) from Graduate University of Chinese Academy of Sciences. Now he is a associate professor in Department of Electronic Information and Electrical Engineering of Changsha University. His current main research interests include time synchronization, satellite navigation and intersatellite links.

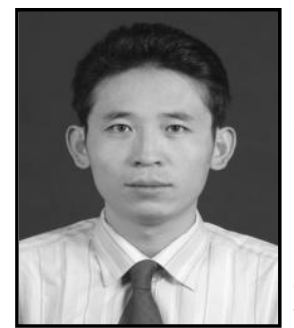

Gun Li. He received his Ph.D. degree in astrometry and celestial mechanics (2007) from Graduate University of Chinese Academy of Sciences. Now he is an associate professor in school of aeronautics and astronautics, university of electronic science and technology of China. His current main research interests include time synchronization, satellite navigation and plasmas physics etc.. 
International Journal of Multimedia and Ubiquitous Engineering

Vol.10, No.5 (2015) 